\title{
The radio luminosity function of cluster radio halos
}

\author{
T. A. Enßlin ${ }^{1}$ and H. Röttgering ${ }^{2}$ \\ 1 Max-Planck-Institut für Astrophysik, Karl-Schwarzschild-Str.1, Postfach 1317, 85741 Garching, Germany \\ 2 Sterrewacht, Oort Gebouw, PO Box 9513, 2300 RA Leiden, The Netherlands
}

Received 19 April 2002 / Accepted 11 September 2002

\begin{abstract}
A significant fraction of galaxy clusters exhibits cluster-wide radio halos. We give a simple prediction of the local and higher redshift radio halo luminosity function (RHLF) on the basis of (i) an observed and a theoretical X-ray cluster luminosity function (XCLF) (ii) the observed radio-X-ray luminosity correlation (RXLC) of galaxy clusters with radio halos (iii) an assumed fraction of $f_{\text {rh }} \approx \frac{1}{3}$ galaxy clusters to have radio halos as supported by observations. We then find 300-700 radio halos with $S_{1.4 \mathrm{GHz}}>1 \mathrm{mJy}$, and $10^{5}-10^{6}$ radio halos with $S_{1.4 \mathrm{GHz}}>1 \mu \mathrm{Jy}$ should be visible on the sky. $14 \%$ of the $S_{1.4 \mathrm{GHz}}>1 \mathrm{mJy}$ and $56 \%$ of the $S_{1.4 \mathrm{GHz}}>1 \mu \mathrm{Jy}$ halos are located at $z>0.3$. Subsequently, we give more realistic predictions taking into account (iv) a refined estimate of the radio halo fraction as a function of redshift and cluster mass, and (v) a decrease in intrinsic radio halo luminosity with redshift due to increased inverse Compton electron energy losses on the Cosmic Microwave Background (CMB). We find that this reduces the radio halo counts from the simple prediction by only $30 \%$ totally, but the high redshift $(z>0.3)$ counts are more strongly reduced by $50-70 \%$. These calculations show that the new generation of sensitive radio telescopes, including LOFAR, ATA, EVLA, SKA and the already-operating GMRT should be able to detect large numbers of radio halos and will provide unique information for studies of galaxy cluster merger rates and associated non-thermal processes.
\end{abstract}

Key words. X-rays: galaxies: clusters - radiation mechanism: non-thermal - radio continuum: general galaxies: intergalactic medium - galaxies: cluster: general

\section{Introduction}

\subsection{Cluster radio halos}

The X-ray emitting intra-cluster medium (ICM) of a significant fraction of galaxy clusters also exhibits cluster-wide radio emission, the so called cluster radio halos (Feretti \& Giovannini 1996; Giovannini et al. 1999b; Kempner \& Sarazin 2001; Giovannini \& Feretti 2000, for recent samples). Cluster radio halos are central, extended over cluster-scales, unpolarised, and steep spectrum radio sources not associated with individual galaxies. It is recognised that radio halos appear in clusters that have recently undergone a major merger event (Tribble 1993; Buote 2001).

While cluster X-ray emission is due to thermal electrons with energies of several $\mathrm{keV}$, the emission of the radio halo is due to synchrotron radiation of relativistic electrons with energies of $\sim 10 \mathrm{GeV}$ in $\sim \mu \mathrm{G}$ magnetic fields. The spatial distribution of the radio emission often seems to follow closely (and nearly linearly) on a large scale the distribution of the $\mathrm{X}$-ray emission (Govoni et al. 2001a). In a few cases, where

Send offprint requests to: T. A. Enßlin, e-mail: ensslin@mpa-garching.mpg.de a cluster merger is in its early stage, detailed observations indicate that the radio halos seem to be spatially restricted to hot merger-shocked regions (Kassim et al. 2001; Markevitch \& Vikhlinin 2001).

The similarity of X-ray and radio morphologies of radio halo galaxy clusters indicates a connection between the energetics of the non-thermal component (magnetic fields and relativistic electrons) and the thermal ICM gas. This is also supported by the strong correlation between radio halo luminosity and the host cluster X-ray luminosity (the RXLC, Liang et al. 2000; Feretti 1999, also see Fig. 1). Since most of the thermal cluster gas is heated in cluster accretion and cluster merger shock waves (e.g. Sunyaev \& Zeldovich 1972; Quilis et al. 1998; Miniati et al. 2000) one would suspect that also the relativistic electrons received their energy from these shocks.

The radiative lifetime of the radio emitting electrons is of the order of 0.1 Gyr (e.g. Jaffe 1977). This is short compared to the shock crossing time in merger events, which is of the order of $1 \mathrm{Gyr}$. If the electrons were accelerated in the shock waves, and just are cooling behind them, the radio emission would not follow the $\mathrm{X}$-ray emission, as observed in late stage merger clusters, but should be more patchy and only located 


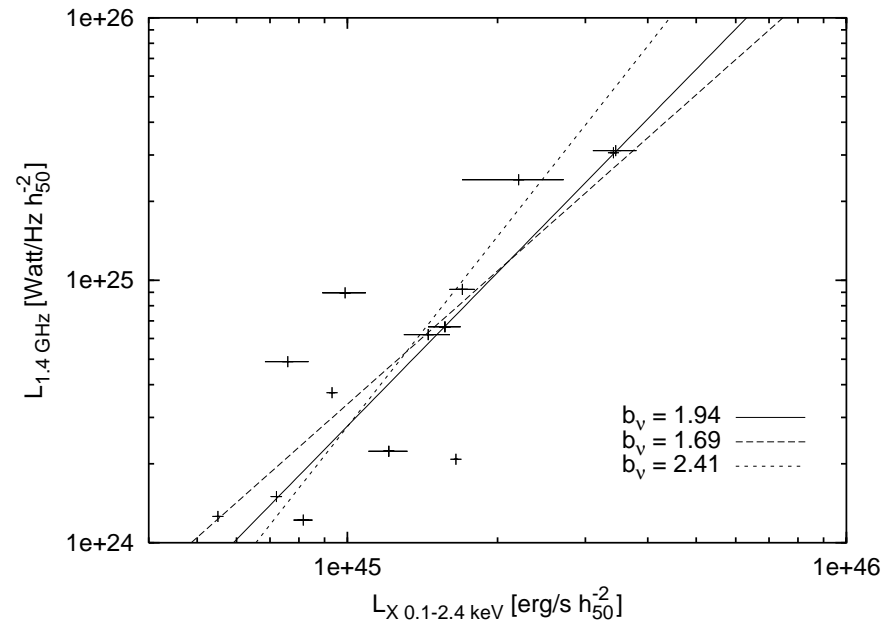

Fig. 1. X-ray and radio luminosity of cluster of galaxies with radio halos. Data is from Feretti (1999) and Govoni et al. (2001b) and the correlation power-laws are given in Sect. 3 .

close to the shock waves ${ }^{1}$. In order to have a radio halo in the post shock region, which lasts sufficiently long to explain the $\mathrm{X}$-ray emission-like morphology of radio halos in later-stage mergers, some fraction of the shock released energy has to be stored in some form and later given to the relativistic radioemitting electron population.

\subsection{Halo formation scenarios}

A suggestion for such an energy storing agent is turbulence within the cluster which may re-accelerate a low energy relativistic electron population against radiative losses (Jaffe 1977, and many others). Such a primary electron model seems to be favoured observationally by spectral index steepening towards higher frequencies as observed in the case of the Coma cluster radio halo (Schlickeiser et al. 1987; Brunetti et al. 2001).

Another suggestion is a shock accelerated population of relativistic protons. Over their long lifetimes they are able to inject the necessary radio-emitting relativistic electrons by charged pion decay after hadronic interactions with the thermal ICM nucleons (Dennison 1980, and others). Such a hadronic scenario for radio halo formation was shown to lead naturally to a very steep RXLC (Colafrancesco 1999; Dolag \& Enßlin 2000; Miniati et al. 2001a), as observed. Such a scenario has - in contrast to the primary models - difficulties in explaining a strong spectral steepening, as seems to be apparent in the Coma cluster (Brunetti 2002). However, measurements of the spectral indices of faint and very extended sources, in the presence of strong point sources, are an observational challenge, so that the possibility of larger uncertainties in the determined radio halo spectra cannot be fully excluded yet. The hadronic

\footnotetext{
1 Such patches of radio emission, the so called cluster radio relics, are indeed observed in merging clusters. They are interpreted to be either emission from shock accelerated ICM electrons (Enßlin et al. 1998; Roettiger et al. 1999; Miniati et al. 2001a) or from shock revived fossil radio cocoons (Enßlin \& Gopal-Krishna 2001; Enßlin \& Brüggen 2002).
}

scenario will soon become further testable since the gamma radiation from the unavoidable neutral pion decay should be detectable by future gamma ray telescopes like GLAST (Vestrand 1982; Enßlin et al. 1997; Colafrancesco \& Blasi 1998; Dolag \& Enßlin 2000; Miniati et al. 2001b).

There are also other suggested radio halo formation scenarios: radio halos were proposed to be superpositions of large numbers of relic radio galaxies (Harris \& Miley 1978, and others), they were proposed to be due to rapidly diffusing electrons escaping from radio galaxies (Holman et al. 1979, and others), and their relativistic electrons were proposed to result from annihilation of neutralinos, if neutralinos are the dominant dark matter component (Colafrancesco \& Mele 2001). Although these are interesting possibilities, they are disfavoured by the apparent association of radio halos with merger shock waves as discussed above.

\subsection{Scientific potential}

In any scenario, cluster radio halos give us deep insight into the physics and properties of galaxy clusters. Very likely, radio halos give a unique probe of non-thermal processes accompanying energetic cluster merger events.

Large numbers of galaxy clusters are expected to be found also at high redshifts by future surveys: e.g. the XMM Large Scale Structure Survey is expected to find $\sim 10^{3}$ galaxy clusters up to redshift one (Refregier et al. 2002), Sunyaev-Zeldovich effect cluster detections with the PLANCK satellite should find $\sim 10^{4}$ galaxy clusters and the Sloan Digital Sky Survey is expected to identify $\sim 5 \times 10^{5}$ clusters (Bartelmann \& White 2002). Using radio halos as tracers of cluster mergers should therefore allow detailed studies of the higher redshift cluster formation processes and properties of the accompanying cluster merger shock waves (Quilis et al. 1998; Miniati et al. 2000). This will be possible due to the strongly increased sensitivity and resolution of the next generation of radio telescopes (e.g. ATA, EVLA, GMRT, LOFAR, SKA). In order to guide the design and observing strategies of these upcoming radio telescopes, predictions for the number of observable radio halos are needed. It is the aim of this paper to provide such predictions, to show their dependence on parameters not yet well constrained, and to indicate their scientific potential.

\subsection{Structure of the paper}

Our predictions are based on (i) estimates of the fraction of clusters containing halos, (ii) the local XCLF and various forms of evolution towards higher redshift, and (iii) the local relation between X-ray and radio halo luminosity of clusters (RXLC).

Having the halo fraction $f_{\mathrm{rh}}$ (Sect. 2) and the RXLC (Sect. 3), the observed present XCLF (Sect. 4) can be translated into the local RHLF (Sect. 5). In order to have predictions for higher redshifts, where the XCLF is not yet measured, we translate a theoretical cluster mass function into an XCLF via a mass-X-ray luminosity correlation (MXLC) of clusters of galaxies (Sect. 4). This also allows predictions of the number counts of cluster radio halos as a function of apparent flux 


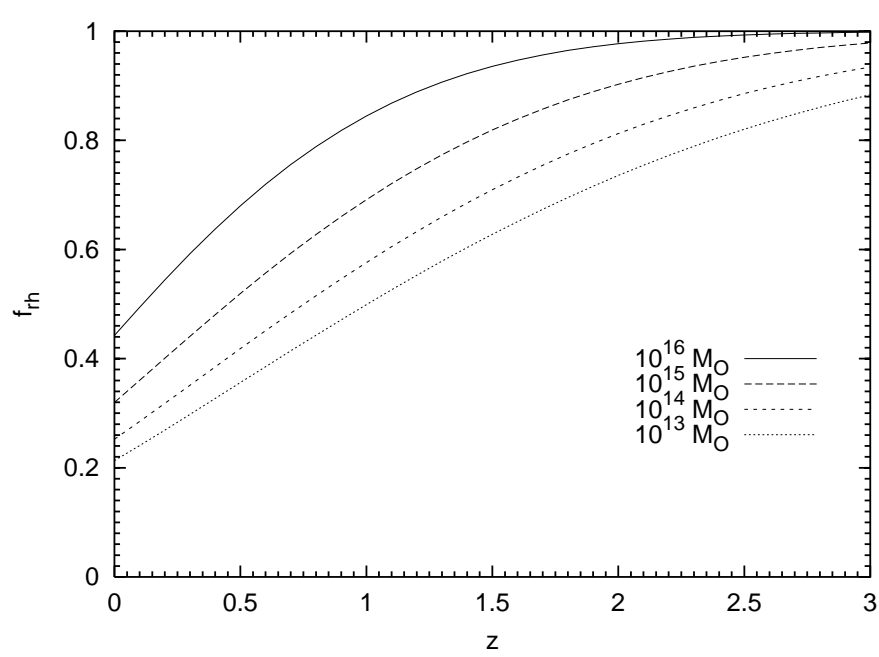

Fig. 2. Fraction of clusters which had a strong mass increase by more than $40 \%$ of their present mass recently (within half a dynamical timescale $\approx 0.09 / H(z)$ ) as a function of their observed redshift and mass in a $\Lambda \mathrm{CDM}$-Universe.

density (Sect. 5). We do this for a constant halo fraction irrespective of cluster mass and redshift, and for one which evolves as the fraction of clusters with recent mergers (Sect. 2). In the latter more realistic calculations we also include a possible dimming effect of halos due to higher radiative losses at higher redshifts. The cluster radio halo detection strategies and expectations are briefly discussed in Sect. 6

Our calculations are done for a $\Lambda$ CDM-Universe with $\Omega_{0}=$ $0.3, \Omega_{\Lambda}=0.7, H_{0}=50 h_{50} \mathrm{~km} \mathrm{~s}^{-1}, \sigma_{8}=0.9$, and $\Gamma=0.21$.

\section{Radio halo fraction}

Giovannini et al. (1999a) find that the detection probability for a radio halo is of the order of $0.3-0.4$ for very X-ray luminous clusters. For less luminous clusters they report a detection probability of as low as 0.05 . Such a low detection rate can arise naturally in a flux limited sample, even if the halo fraction is much higher, if most of the sources are below the detection limit. In our case, the low X-ray luminosity clusters are also expected to contain the weakest radio halos (see Fig. 3), which are therfore expected to be most likely missed in sensitivitylimited radio observations. Thus we feel that this low number of radio halos in low X-ray luminosity clusters is likely due to a selection effect naturally arising in searches for radio halos with luminosities close to or below the frontier of observational feasibility.

Here, we assume that every cluster that recently grew strongly exhibits a radio halo. Thus we implicitly assume that the radio halo energy release is somehow delayed after the cluster merger shock passage, as discussed in the introduction. As a crude rule of thumb we adopt a constant value of $f_{\mathrm{rh}}=\frac{1}{3}$ for the fraction of clusters with a radio halo as indicated by observations of high X-ray luminosity clusters. This number is smaller than the number of cluster of clusters exhibiting substructure (40\%-60\%, e.g. Mohr et al. 1995; Jones \& Forman 1999; Schuecker et al. 2001), but only large merger seem to produce radio halos (Buote 2001).
This number can also be estimated with the help of the extended Press-Schechter formalism ${ }^{2}$, and thereby extrapolated to different cluster mass ranges and higher redshifts. If one assumes that all clusters which had a mass increase of more than $40 \%$ of their final mass within half a dynamical timescale of the final cluster (which is approximately $\Delta t \approx 0.09 / H(z)$ with $H(z)$ the Hubble parameter at redshift $\mathrm{z}$ ) exhibit a radio halo, one finds that $f_{\mathrm{rh}}=0.32$ for present day clusters with a mass of $10^{15} M_{\odot}$. The resulting halo fraction is displayed in Fig. 2 as a function of redshift and cluster mass.

In an earlier study Fujita \& Sarazin (2001) made a similar calculation of the cluster merger rates. Their estimate of the fraction of cluster radio halos is based on the radiative energy loss timescale of the radio electrons. Since this is much shorter than the dynamical timescale of the clusters used in our work, their fraction of galaxy clusters exhibiting radio halos is much smaller. They find that only $10 \%$ of the present clusters had a major merger recently enough (within the electron cooling time) to exhibit a radio halo. In order to reproduce the fraction of $20 \%-30 \%$ of all present clusters, which is indicated by observations, they require that rather weak mergers with a mass increase of only $10 \%$ have to be sufficient to trigger a radio halo. In contrast to this, clusters with radio halos exhibit signatures of much stronger merging activity than a $10 \%$ mass increase would produce ${ }^{3}$. This indicates that the relevant timescale of halo emission after a merger should be significantly longer than the electron cooling timescale, and is likely to be of the order of the dynamical timescale of the merger as assumed in our work.

${ }^{2}$ We use Eq. (2.26) in Lacey \& Cole (1993) to estimate the conditional probability that a cluster of given mass $M_{2}$ at redshift $z_{2}$ had a progenitor which was more massive than $0.6 M_{2}$ at an earlier redshift $z_{1}$, which gives us the fraction of clusters without recent strong merging $1-f_{\text {rh }}$. Contrary to a statement in Lacey \& Cole (1993), van den Bosch (2002) demonstrates that this formula gives an accurate estimate of the extended Press-Schechter prediction of this probability. Compared to numerical CDM simulations the agreement is worse, but acceptable for our purpose. We use the critical overdensity parameter $\delta_{\mathrm{c}, 0}(z)$ and the mass variance of the smoothed density field $\sigma(M)$ in the parametrization given in van den Bosch (2002) and adopt a $\Lambda$ CDM cosmology as defined in the introduction.

3 This can be seen by the fact that only clusters with strong substructure have halos (Buote 2001). That even a $20 \%$ merger is insufficient to trigger a radio halo is also demonstrated by the observations of the cluster Abell 3667. It has signatures of a recent merger since substructure exists and two giant cluster radio relics indicate the presence of peripheral shock waves (Röttgering et al. 1997; Enßlin et al. 1998, and references therein). A detailed numerical simulation of this cluster by Roettiger et al. (1999) showed that its X-ray morphology is reproduced well by an on-axis collision of two clusters with a mass ratio of 1:5. Although the merger in Abell 3667 is fully developed no radio halo could be found in the sensitive radio observations (Röttgering et al. 1997). The reason for this is likely that the cluster centre was not shocked in this weak merger, as the presence of a cool core demonstrates (Vikhlinin et al. 2001). On the other hand, a merger with a mass ratio of 1:2.5 seems to be sufficiently violent to trigger a radio halo, as the cluster Abell 754 suggests (Roettiger et al. 1998; Kassim et al. 2001). 


\section{Radio halo-X-ray luminosity correlation}

Feretti (1999) compiled the properties of the presently known cluster radio halos and cluster radio relics. In the following we use the properties of the cluster radio halo sub-sample listed in this work, plus the properties of the radio halo of Abell 2254, which we take from Govoni et al. (2001b).

In Fig. 1 we show the $0.1-2.4 \mathrm{keV} \mathrm{X}$-ray and $1.4 \mathrm{GHz}$ radio luminosities of the galaxy clusters containing radio halos. Also shown are power-law fits of the form

$L_{v}\left(L_{\mathrm{X}}\right)=a_{v} 10^{24} h_{50}^{-2}$ Watt $/ \mathrm{Hz}\left(\frac{L_{X}}{10^{45} h_{50}^{-2} \mathrm{erg} / \mathrm{s}}\right)^{b_{v}}$.

We obtain the parameters $a_{v}=3.37$ and $b_{v}=1.69$ from linear regression in $\log$ arithmic units with $\log \left(L_{v}\right)$ as the dependent variable. If $\log \left(L_{X}\right)$ is assumed to be the dependent variable we get $a_{v}=2.77$ and $b_{v}=2.41$. A fit using errors in both observables (assuming an uncertainty of $\Delta L_{v} / L_{v}=0.1$ ) yields $a_{v}=2.78$ and $b_{v}=1.94$. We use all three parameter sets to calculate the local RHLF, but favour the latter parameters with intermediate slope since the other slopes are likely affected by the scatter in the data.

It should be noted that the fitted RXLCs are used throughout this paper to extrapolate halo properties for lower and higher luminosities than yet observationally constrained. The underlying idea is that several of the proposed scenarios for radio halo formation discussed in the introduction predict such or similar scaling relations. At the moment, this extrapolation is therefore only an educated guess, which should be tested by more sensitive future observations.

There might be an additional redshift dependence of the radio halo luminosity. For our models with constant halo fraction we do not assume any redshift dependence of the RXLC in order to keep the model simple. For the more realistic scenario with evolving halo fraction we assume $L_{v}\left(L_{\mathrm{X}}, z\right)=L_{v}\left(L_{\mathrm{X}}\right)(1+z)^{-4}$, since for weak cluster magnetic fields $(B \leq \mu \mathrm{G})$ the ratio of synchrotron to total (synchrotron plus inverse Compton) energy losses is proportional to the inverse $\mathrm{CMB}$ photon energy density. If the typical cluster magnetic field energy densities are comparable or even stronger than the CMB energy density, this approach underestimates the radio halo luminosity. Hence, by including it we give a conservative estimate.

\section{X-ray cluster luminosity function}

The XCLF in the ROSAT 0.1-2.4 keV band was recently estimated by Böhringer et al. (2002). They fit their data by

$$
\frac{\mathrm{d} N_{\mathrm{cl}}}{\mathrm{d} L_{\mathrm{X}}}=\frac{n_{\mathrm{cl}}}{L_{\mathrm{X}, *}}\left(\frac{L_{\mathrm{X}}}{L_{\mathrm{X}, *}}\right)^{-\alpha_{\mathrm{X}}} \exp \left(-\frac{L_{\mathrm{X}}}{L_{\mathrm{X}, *}}\right),
$$

with $L_{\mathrm{X}, *}=8.36 \times 10^{44} h_{50}^{-2} \mathrm{erg} / \mathrm{s}, n_{\mathrm{cl}}=107\left(\mathrm{Gpc} / h_{50}\right)^{-3}$, and $\alpha_{\mathrm{X}}=1.69$ for a $\Lambda \mathrm{CDM}$ cosmology (see Fig. 3 ).

To be able to extrapolate this locally determined XCLF to higher redshifts, we take a two-step approach. First, we relate a halo mass to an individual X-ray luminosity. Second, the models for the growth of the halo masses with time then naturally give the evolution of the XCLF.

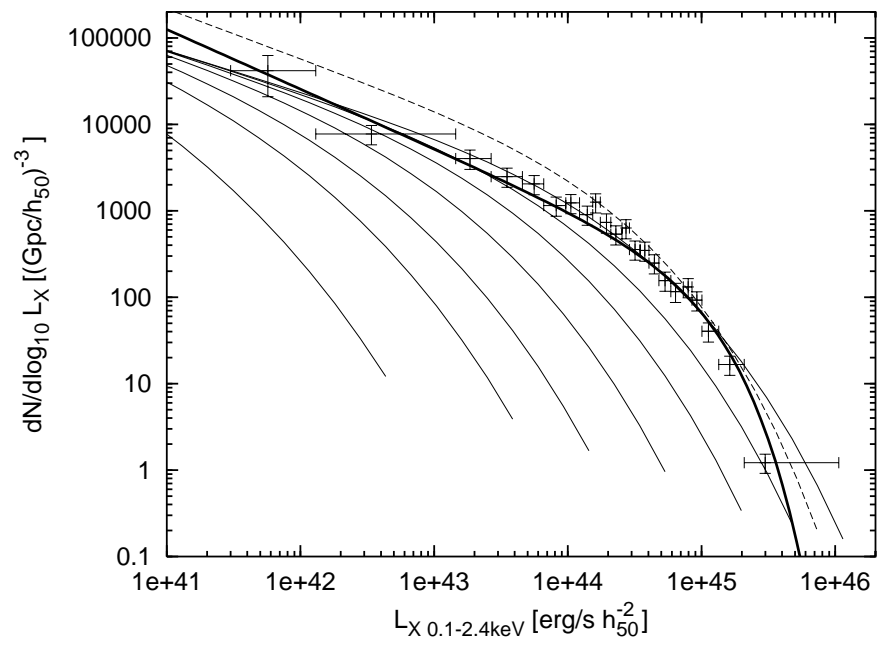

Fig. 3. X-ray luminosity function. Data points and thick line are from Böhringer et al. (2002). The dashed line is the Jenkins et al. (2001) mass function translated with the help of the Reiprich \& Böhringer (2002) MXLC. The thin solid lines are the same mass functions translated with our adapted MXLC for redshift $z=0,0.3,0.6,1.0,1.5,2.0$, and 3.0 from top to bottom. These lines end where the range of the simulated mass functions end.

We translate the cluster mass $\left(M_{200, \mathrm{~J}}\right.$, "J" stands for Jenkins, etc.) function of Jenkins et al. (2001) into an X-ray luminosity function. This can be done with the empirical MXLC of Reiprich \& Böhringer (2002), which is based on hydrostatic cluster mass estimates ${ }^{4}$ :

$L_{\mathrm{X}}=a_{\mathrm{X}} 10^{45} h_{50}^{-2} \mathrm{erg} / \mathrm{s}\left(\frac{M_{200, \mathrm{R} \& \mathrm{~B}}}{10^{15} h_{50}^{-1} M_{\odot}}\right)^{b_{\mathrm{X}}}$,

where $a_{\mathrm{X}}=0.511$ and $b_{\mathrm{X}}=1.571$ (for their BCSE-Bisector fit of their extended sample). The resulting local XCLF significantly deviates from the observed one ${ }^{5}$ (see Fig. 3), and we therefore do not use it any further.

In order to have a working model XCLF we adopt the mass function of Jenkins et al. (2001) and re-fitted the parameters in Eq. (3) so that the measured local XCLF is reproduced within the sampled range (see Fig. 3). This gives $a_{\mathrm{X}}=0.449$ and $b_{\mathrm{X}}=1.9$, which we adopt in the following and denote it as our adapted MXLC (note that we insert $M_{200, \mathrm{R} \& \mathrm{~B}} \approx M_{200, \mathrm{~J}} \Omega_{0}^{1 / 2}$ in Eq. (3) in order to correct for the difference in the definitions of the cluster masses).

Our model predicts some evolution of the high luminosity end of the XCLF at moderate redshifts $(0<z<1)$. This may be in conflict with measurements of the higher redshift XCLF,

\footnotetext{
${ }^{4}$ Care has to be taken even though both works give $M_{200}$, the mass contained in a region with an overdensity of a factor 200 , since Reiprich \& Böhringer (2002) refer to the critical density $\varrho_{c}$, whereas Jenkins et al. (2001) refer to the cosmic mean density $\varrho_{0}=\Omega_{0} \varrho_{\mathrm{c}}$. We correct for this by using $M_{200, \mathrm{R} \& \mathrm{~B}} \approx M_{200, \mathrm{~J}} \Omega_{0}^{1 / 2}$, which is exact for a singular isothermal sphere and therefore acceptable for the large cluster radii involved.

5 This may be caused by a different set of cosmological parameters (a better agreement with a $\Lambda$ CDM Universe with $\Omega_{0}=0.12$ and $\sigma_{8}=0.96$ was found by Reiprich \& Böhringer 2002), or by systematic uncertainties in the underlying hydrostatic mass estimates.
} 


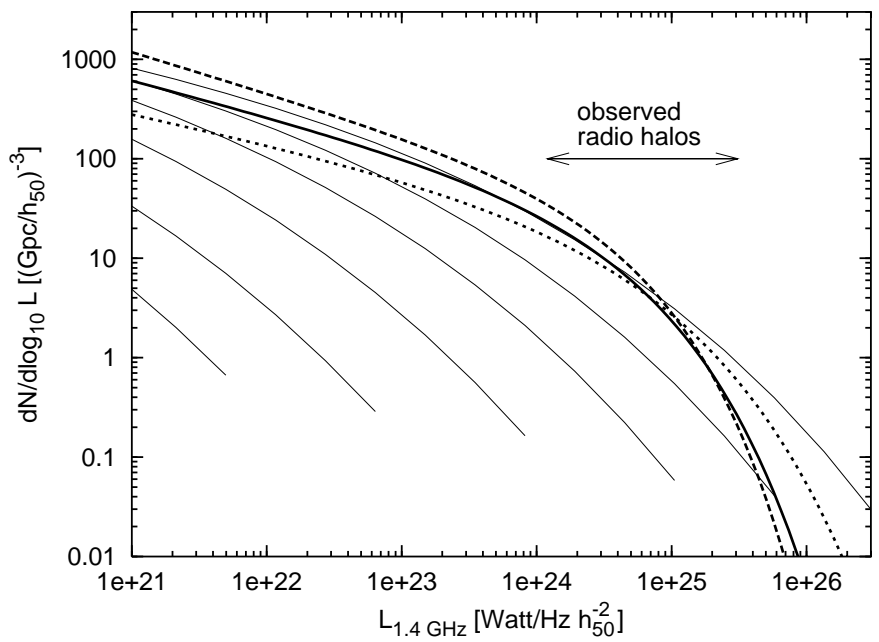

Fig. 4. Radio halo luminosity function, derived under the assumption that a constant fraction $f_{\mathrm{rh}}=1 / 3$ of all clusters contain a radio halo. The thick lines are calculated from the observed X-ray luminosity function, which was translated using the correlations displayed in Fig. 1. The thin solid lines are calculated from our adapted X-ray luminosity function using the intermediate steep correlation displayed in Fig. 1 for redshift $z=0,0.3,0.6,1.0,1.5,2.0$, and 3.0 from top to bottom. These lines end where the range of the underlying simulated mass functions end.

which do not reveal very significant evolution of the XCLF in this redshift range (de Grandi et al. 1999, for a discussion). On the other hand, the error bars of these measurements are still quite large and could be consistent with the amount of evolution given in our model. In order to also cover the possible case that there is no evolution in the XRF in the redshift range most important for the radio halo source counts, we also present calculations in which the local XCLF is assumed to hold at all redshifts. This gives a much larger number of radio halos. Thus our evolving XRF model can be regarded to be conservative since it may underpredict the number of luminous clusters in $\mathrm{X}$-ray and radio.

\section{Radio halo luminosity function}

The local RHLF follows from Eqs. (1) and (2):

$\frac{\mathrm{d} N_{\mathrm{rh}}}{\mathrm{d} L_{v}}=\frac{n_{\mathrm{rh}}}{L_{v, *}}\left(\frac{L_{v}}{L_{v, *}}\right)^{-\alpha_{\mathrm{rh}}} \exp \left(-\left[\frac{L_{v}}{L_{v, *}}\right]^{\beta_{\mathrm{rh}}}\right)$,

with $n_{\mathrm{rh}}=f_{\mathrm{rh}} n_{\mathrm{cl}} / b_{v}, L_{v, *}=L_{v}\left(L_{\mathrm{X}, *}\right), \alpha_{\mathrm{rh}}=\left(\alpha_{\mathrm{X}}+b_{v}-1\right) / b_{v}$, and $\beta_{\mathrm{rh}}=1 / b_{v}$. This is displayed for the different RXLCs in Fig. 4 together with the RHLF computed from our adapted XCLF and the intermediate steep RXLC.

In order to be able to calculate the radio halo number counts, we fit the adapted RHLF by a functional form like Eq. (4) separately for various redshifts. This allows to extrapolate to higher radio halo luminosities and therefore to calculate the flux density distribution by integration over the evolving radio halo population. We assume a common radio halo spectral index of $\alpha_{v}=1$ for this.

The resulting flux density distribution (Fig. 5) should depend little on cosmology for larger fluxes. This is because it

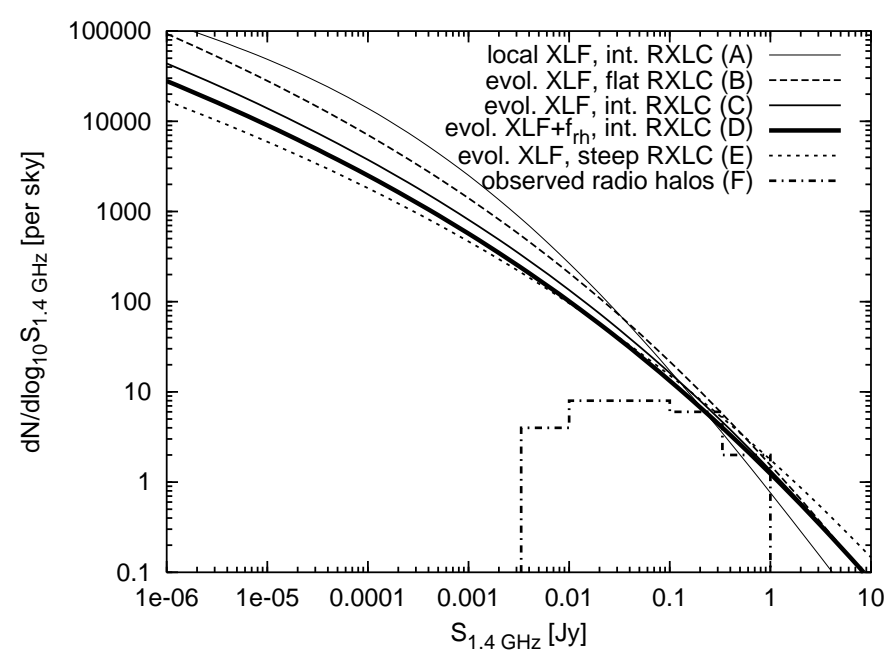

Fig. 5. Expected flux density ditribution of radio halos. All solid lines show models using the intermediate radio-X-ray luminosity correlation. The thin solid line (A) shows the flux density distribution if the local intermediate radio halo luminosity function (thick solid line in Fig. 4) is assumed to hold at all redshifts. The heavy solid line (D) uses the adapted X-ray luminosity function, but assumes that the fraction of clusters with radio halos are the one with recent mergers as displayed in Fig. 2. In addition a $(1+z)^{-4}$ decline in radio luminosity is assumed in that latter model as a consequence of the higher inverse Compton energy losses on the CMB at higher redshift. The lines B, $\mathrm{C}$, and $\mathrm{E}$ result from our adapted model, using the three radio-X-ray luminosity correlations (RXLC) displayed in Fig. 1. Finally, the histogram (F) shows the flux density distribution of the cluster sample compiled by Feretti (1999) (plus A2254 from Govoni et al. 2001b).

is dominated at the bright end by the local RHLF, which was fixed by observational constraints. In order to illustrate this, Fig. 5 also contains the flux density distribution calculated by using the local RHLF given by Eq. (4) for all redshifts. Also included in this figure are more realistic calculations including a non-constant halo fraction as displayed in Fig. 2 and dimming at higher redshifts. For the more realistic scenario (evolving XCLF, evolving $f_{\mathrm{rh}}$, redshift dimming) the contributions of different redshift ranges to the flux density distribution is displayed in Fig. 6. Further, we have included in both figures a histogram with the observed flux density distribution of the radio halo sample of Feretti (1999) (plus A2254 from Govoni et al. 2001b). The large discrepancy between the observed and expected flux density distribution indicates a large incompleteness of our present knowledge of faint cluster radio halos.

The redshift distribution of radio halos above given flux limits is displayed in Fig. 7 for the most realistic model. A comparison with the already observed population of radio halos shows that the number of higher redshift clusters $(0.3<z<0.6)$ observed is close to the predictions. This means that either observers already managed to find a substantial fraction of these halos or that our most realistic model is indeed too conservative. If redshift dimming would not occur, e.g. because cluster magnetic fields are strong and therefore the CMB is not the main energy loss target of the electrons, then many more higher redshift radio halos are expected as also shown in Fig. 7. 
Table 1. The number $N$ of expected radio halos on the full sky, which are above a given flux density $S_{1.4 \mathrm{GHz} \text {,min }}$ for the flat ( $N^{\text {flat }}$ ), the intermediate $\left(N^{\text {int. }}\right)$, and the steep $\left(N^{\text {steep }}\right)$ radio halo-X-ray luminosity correlations displayed in Fig. 1 . In addition to the model with an evolving X-ray luminosity function $\left(N_{\text {evol. }}\right.$, see Fig. 5) also the radio halo number counts for a redshift independent (=local) cluster distribution are given $\left(N_{\text {local }}\right.$, see Fig. 5$)$ for the intermediate RXLC. Further, the models marked by $*$ give the expected number counts assuming that the fraction of clusters with radio halos is not $f_{\mathrm{rh}}=\frac{1}{3}$ as assumed in the other calculations, but is given by the fraction of clusters which had a recent strong mass increase, as displayed in Fig. 2. In addition to this, it is assumed that the radio halo luminosity of a cluster with the same mass is lower by a factor $(1+z)^{-4}$ due to the increasing inverse Compton energy losses on the CMB. Thus, the first three columns indicate the level of uncertainty in these calculations due to the uncertainty in the RXLC, Cols. 4 and 5 give an optimistic model, and the last two columns give the most likely estimate.

\begin{tabular}{rrrrrrrrr}
\hline \hline \multicolumn{2}{c}{$S_{1.4 \mathrm{GHz}, \text { min }}$} & $N_{\text {evol. }}^{\text {flat }}$ & $N_{\text {evol. }}^{\text {int. }}$ & $N_{\text {evol. }}^{\text {steep }}$ & $N_{\text {local }}^{\text {int. }}$ & $N_{\text {local }}^{\text {int, }}$ & $N_{\text {evol. }}^{\text {int., }}$ & $N_{\text {evol. }}^{\text {int. }}(z>0.3)$ \\
\hline 1 & $\mu \mathrm{Jy}$ & 74857.9 & 36646.9 & 15388.6 & 118854.0 & 70579.6 & 23758.5 & 10784.9 \\
10 & $\mu \mathrm{Jy}$ & 19784.7 & 10269.5 & 4821.5 & 36733.0 & 19686.2 & 6812.2 & 2123.7 \\
100 & $\mu \mathrm{Jy}$ & 4308.1 & 2403.8 & 1298.1 & 8076.4 & 4247.7 & 1653.5 & 280.9 \\
1 & $\mathrm{mJy}$ & 735.7 & 450.2 & 290.6 & 1143.8 & 664.7 & 326.4 & 20.5 \\
10 & $\mathrm{mJy}$ & 93.3 & 64.2 & 52.0 & 100.0 & 71.0 & 50.1 & 0.6 \\
100 & $\mathrm{mJy}$ & 8.4 & 6.7 & 7.1 & 5.6 & 5.0 & 5.7 & 0.0 \\
1 & $\mathrm{Jy}$ & 0.5 & 0.5 & 0.7 & 0.2 & 0.2 & 0.5 & 0.0 \\
\hline
\end{tabular}

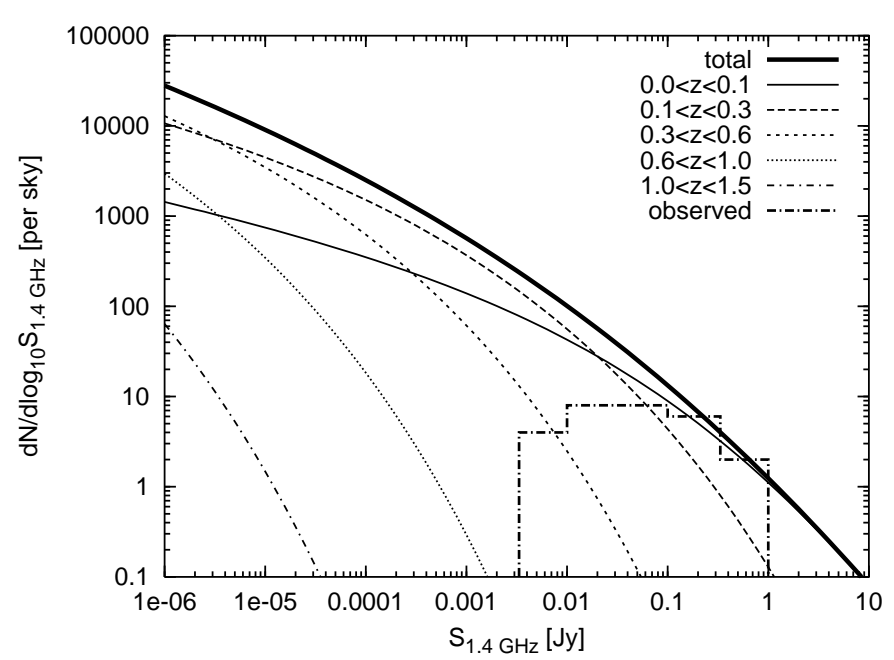

Fig. 6. Expected flux density distribution of radio halos for the most realistic model (see Fig. 5 for a comparison). The smooth curves below give the radio halo flux density distribution for restricted redshift ranges as indicated in the figure. The histogram shows the flux density distribution of the cluster sample compiled by Feretti (1999) (plus A2254 from Govoni et al. 2001b) which contains clusters in the redshift range up to $z=0.55$.

\section{Discussion}

We estimated the cluster radio halo luminosity function and the expected flux density distribution by translating an observed and a theoretical X-ray cluster luminosity function with the help of the observed cluster radio halo-X-ray luminosity correlation. A power-law form of this correlation was used to extrapolate into the observationally poorly constraint regime of (weak) radio halos of low X-ray luminosity clusters. For a simple model calculation we assumed that a fraction $f_{\mathrm{rh}}=\frac{1}{3}$ of all clusters contain radio halos, irrespective of redshift and cluster size. We note, that if the halo fraction for low X-ray luminosity clusters would be much lower, which cannot be excluded with the present day data, our predictions based on the above halo

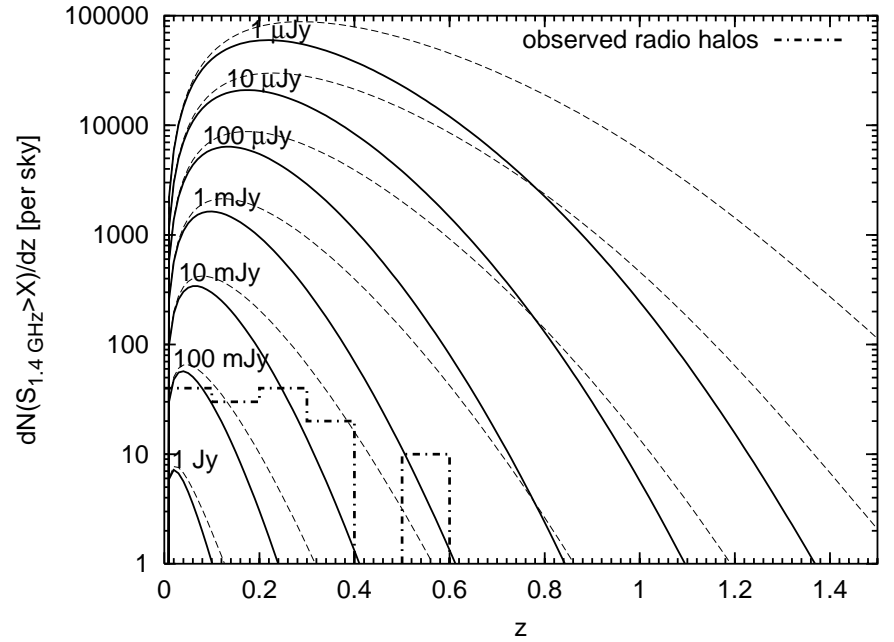

Fig. 7. Expected redshift distribution of radio halos with fluxes above flux limits as indicated in the figure. The solid lines give the most realistic model, whereas the dashed lines do not include any radio halo dimming with redshift. The histogram shows the differential redshift distribution of the radio halo cluster sample compiled by Feretti (1999) and Govoni et al. (2001b) (binned into bins of width $\Delta z=0.1$ ).

fraction would be overestimated. In the case that the halo fraction is the same for all cluster, but lower than assumed here, our results can simply be re-scaled.

The above assumptions may be questioned, since both the higher merging rate of clusters of galaxies and also the increased electron inverse Compton losses at higher redshifts can modify the fraction of clusters having radio halos. For that reasons also calculations were presented in which we tried to take both effects into account. If our assumptions hold, we are able to predict the number of detectable radio halos with upcoming sensible radio telescopes like LOFAR, ATA, EVLA, SKA, and also the existing GMRT. Detailed numbers for the different models can be found in Table 1.

The LOFAR array as an example: the point source sensitivity at $120 \mathrm{MHz}$ is expected to be $0.13 \mathrm{mJy}$ within 1 hour 
integration time and a $4 \mathrm{MHz}$ bandwidth. A survey covering half of the sky can be accomplished in a years timescale at this frequency and with this depth. It would find $800-1200_{-40 \%}^{+80 \%}$ radio halos ${ }^{6}$ with a significance of 10 sigma, sufficient for further follow up observations. Within this sample $140-300_{-40 \%}^{+80 \%}$ of the radio halos are expected to have redshifts larger than 0.3 .

A more efficient strategy to find cluster radio halos would be to use the large future cluster catalogues from SDSS, PLANCK, and XMM-Newton as a target list for deep integrations with the upcoming sensitive radio telescopes. This should allow tests of many of the hypotheses (partly used in this work) on redshift and cluster size dependencies of the radio halo population, helping to establish cluster radio halos as a tool to investigate galaxy cluster formation and the non-thermal processes accompanying it.

Acknowledgements. This work benefited from discussions with $\mathrm{M}$. Bartemann, H. Böhringer, H. Matthis, F. Miniati, P. Schuecker, F. van den Bosch, S. D. M. White, and from comments of an anonymous referee. We have made use of the mass function program of Jenkins et al. (2001). TAE thanks the LOFAR collaboration for the invitation and the financial support to participate in the LOFAR workshop at the Haystack Observatory (2001) where this work was initiated. This work was done in the framework of the EC Research and Training Network The Physics of the Intergalactic Medium.

\section{References}

Böhringer, H., Collins, C. A., Guzzo, L., et al. 2002, ApJ, 566, 93 Bartelmann, M., \& White, S. D. M. 2002, A\&A, 388, 732

Brunetti, G. 2002, in Matter and Energy in Clusters of Galaxies, ed. S. Bowyer, \& C.-Y. Hwang, ASP Conf. Ser., in preparation [astro-ph/0208074]

Brunetti, G., Setti, G., Feretti, L., \& Giovannini, G. 2001, MNRAS, 320,365

Buote, D. A. 2001, ApJ, 553, L15

Colafrancesco, S. 1999, in Ringberg Workshop on Diffuse Thermal and Relativistic Plasma in Galaxy Clusters, ed. P. S. H. Böhringer, \& L. Feretti, 271 of MPE Report, 269

Colafrancesco, S., \& Blasi, P. 1998, Astropart. Phys., 9, 227

Colafrancesco, S., \& Mele, B. 2001, ApJ, 562, 24

de Grandi, S., Guzzo, L., Böhringer, H., et al. 1999, ApJ, 513, L17

Dennison, B. 1980, ApJ, 239, L93

Dolag, K., \& Enßlin, T. A. 2000, A\&A, 362, 151

Enßlin, T. A., Biermann, P. L., Klein, U., \& Kohle, S. 1998, A\&A, 332, 395

Enßlin, T. A., Biermann, P. L., Kronberg, P. P., \& Wu, X.-P. 1997, ApJ, 477,560
Enßlin, T. A., \& Brüggen, M. 2002, MNRAS, 331, 1011

Enßlin, T. A., \& Gopal-Krishna, 2001, A\&A, 366, 26

Feretti, L. 1999, in The Universe at Low Radio Frequencies, IAU Symp. 199 [astro-ph/0006379]

Feretti, L., \& Giovannini, G. 1996, in Extragalactic Radio Sources, IAU Symp., 175, 333

Fujita, Y., \& Sarazin, C. L. 2001, ApJ, 563, 660

Giovannini, G., \& Feretti, L. 2000, New Astron., 5, 335

Giovannini, G., Feretti, L., \& Govoni, F. 1999a, in The Universe at Low Radio Frequencies, IAU Symp. 199 [astro-ph/0006380]

Giovannini, G., Tordi, M., \& Feretti, L. 1999b, New Astron., 4, 141

Govoni, F., Enßlin, T. A., Feretti, L., \& Giovannini, G. 2001a, A\&A, 369,441

Govoni, F., Feretti, L., Giovannini, G., et al. 2001b, A\&A, 376, 803

Harris, D. E., \& Miley, G. K. 1978, A\&AS, 34, 117

Holman, G. D., Ionson, J. A., \& Scott, J. S. 1979, ApJ, 228, 576

Jaffe, W. J. 1977, ApJ, 212, 1

Jenkins, A., Frenk, C. S., White, S. D. M., et al. 2001, MNRAS, 321, 372

Jones, C., \& Forman, W. 1999, ApJ, 511, 65

Kassim, N. E., Clarke, T. E., Enßlin, T. A., Cohen, A. S., \& Neumann, D. M. 2001, ApJ, 559, 785

Kempner, J. C., \& Sarazin, C. L. 2001, ApJ, 548, 639

Lacey, C., \& Cole, S. 1993, MNRAS, 262, 627

Liang, H., Hunstead, R. W., Birkinshaw, M., \& Andreani, P. 2000, ApJ, 544, 686

Markevitch, M., \& Vikhlinin, A. 2001, ApJ, 563, 95

Miniati, F., Jones, T. W., Kang, H., \& Ryu, D. 2001a, ApJ, 562, 233

Miniati, F., Ryu, D., Kang, H., \& Jones, T. W. 2001b, ApJ, 559, 59

Miniati, F., Ryu, D., Kang, H., et al. 2000, ApJ, 542, 608

Mohr, J. J., Evrard, A. E., Fabricant, D. G., \& Geller, M. J. 1995, ApJ, 447,8

Quilis, V., Ibanez, J. M. A., \& Saez, D. 1998, ApJ, 502, 518

Refregier, A., Valtchanov, I., \& Pierre, M. 2002, A\&A, 390, 1

Reiprich, T. H., \& Böhringer, H. 2002, ApJ, 567, 716

Roettiger, K., Burns, J. O., \& Stone, J. M. 1999, ApJ, 518, 603

Roettiger, K., Stone, J. M., \& Mushotzky, R. F. 1998, ApJ, 493, 62

Röttgering, H. J. A., Wieringa, M. H., Hunstead, R. W., \& Ekers, R. D. 1997, MNRAS, 290, 577

Schlickeiser, R., Sievers, A., \& Thiemann, H. 1987, A\&A, 182, 21

Schuecker, P., Böhringer, H., Reiprich, T. H., \& Feretti, L. 2001, A\&A, 378, 408

Sunyaev, R. A., \& Zeldovich, Y. B. 1972, A\&A, 20, 189

Tribble, P. C. 1993, MNRAS, 263, 31

van den Bosch, F. C. 2002, MNRAS, 331, 98

Vestrand, W. T. 1982, AJ, 87, 1266

Vikhlinin, A., Markevitch, M., \& Murray, S. S. 2001, ApJ, 551, 160

\footnotetext{
${ }^{6}$ The first (lower) number result from our most realistic model, the second (higher) from the model with constant $f_{\text {rh }}$ and constant RXLC; the error range indicates the uncertainties resulting from the possible slopes of the RXLC; a radio halo spectral index of $\alpha_{v}=1$, which is a conservative assumption for this purpose, was used in the frequency interpolation.
} 